\title{
REVERSÃO DE ANSIEDADE À MATEMÁTICA: ALGUNS DADOS DA LITERATURA ${ }^{1}$
}

João dos Santos Carmo*

Aline Morales Simionato"

\begin{abstract}
RESUMO. A ansiedade em relação à matemática é um fenômeno que compreende reações emocionais negativas diante de situações que requisitam o uso de conhecimentos matemáticos. É particularmente identificada no contexto escolar e está diretamente relacionada a experiências inadequadas de ensino dessa disciplina. O objetivo do presente artigo é fornecer um panorama atualizado da literatura que trata especificamente de um fenômeno chamado de ansiedade em relação à matemática. Inicialmente serão expostos a definição e os conceitos principais relacionados ao fenômeno em foco e, em seguida, alguns estudos acerca da reversão da ansiedade em relação à matemática. Por fim, serão apontadas algumas implicações para a pesquisa e para o desenvolvimento de programas brasileiros que visam ajudar indivíduos que apresentem ansiedade em relação à matemática.
\end{abstract}

Palavras-chave: Ansiedade em relação à Matemática; controle aversivo; reversão de ansiedade em relação à matemática.

\section{REVERSION OF MATHEMATICS ANXIETY: SOME DATA FROM LITERATURE}

\begin{abstract}
Mathematics anxiety is a phenomenon composed by negative emotional reactions in situations that require the use of mathematics knowledge. It is particularly present in school context, and is directly related to inadequate teaching experiences of that subject matter. The present article aims to convey an up to date panorama of the literature that leads specifically with a phenomenon known as mathematics anxiety. Initially, there will be exposed the definition and the main concepts related to the phenomenon as well as some studies about the reversion of mathematics anxiety. Finally, some implications to future researches and to the development of Brazilian programs to help individuals with mathematics anxiety are pointed out.
\end{abstract}

Key words: Mathematics anxiety; aversive control; reversion of mathematics anxiety.

\section{REVERSIÓN DE ANSIEDAD A LAS MATEMÁTICAS: ALGUNOS DATOS DE LA LITERATURA}

\begin{abstract}
RESUMEN. La ansiedad a las matemáticas es un fenómeno compuesto por reacciones emocionales negativas delante de situaciones que requieren el uso de conocimientos matemáticos. Es particularmente reconocida en el contexto escolar y está directamente relacionada a experiencias inadecuadas de enseñanza de esa disciplina. El objetivo de este artículo es ofrecer una visión actualizada de la literatura que trata específicamente de un fenómeno llamado ansiedad a las matemáticas. Inicialmente serán presentados la definición y los principales conceptos relacionados con el fenómeno en foco y, a continuación, algunos estudios sobre la reversión de la ansiedad a las matemáticas. Finalmente, serán señaladas algunas implicaciones para la investigación y para el desarrollo de programas brasileños destinados a ayudar a las personas que presentan ansiedad a las matemáticas.
\end{abstract}

Palabras-clave: Ansiedad a las matemáticas; control aversivo; remediación de ansiedad a las matemáticas.

Apoio: CNPq.

Doutor em Educação pela Universidade Federal de São Carlos (UFSCar). Professor do Departamento de Psicologia da UFSCar e do Programa de Pós-Graduação em Psicologia da UFSCar. Pesquisador do Instituto Nacional de Ciência e Tecnologia sobre Comportamento, Cognição e Ensino (INCT-ECCE). Líder do Grupo de Pesquisa Análise do Comportamento e EnsinoAprendizagem da Matemática (ACEAM), Diretório dos Grupos de Pesquisa do CNPq.

\# Graduanda em Psicologia pela Universidade Federal de São Carlos. Bolsista de Iniciação Científica pelo Programa PIBIC/CNPq. Membro do Grupo de Pesquisa Análise do Comportamento e Ensino-Aprendizagem da Matemática (ACEAM) registrado no Diretório dos Grupos de Pesquisa - CNPq. 
Um estudo seminal, conduzido por Dreger e Aiken (1957), identificou que muitos estudantes universitários relatavam desconforto diante de situações que envolviam a resolução de problemas de matemática. Dreger e Aiken passaram a chamar a essas reações de "ansiedade a números" e relataram que os estudantes não apresentavam ansiedade generalizada, mas um tipo de ansiedade específica ao uso e aplicação de cálculos e outras relações numéricas. A expressão "ansiedade a números" foi substituída por "ansiedade matemática" "2 por Tobias (1978), por ser uma expressão mais condizente com os relatos que passaram a se multiplicar, envolvendo estudantes dos diversos níveis de ensino e também indivíduos que já haviam passado pelos anos escolares.

Estados de ansiedade envolvem reações fisiológicas relatadas como desagradáveis, postura tensa; expressão facial cansada; movimentos sem direção; dores de cabeça; distúrbios estomacais; mãos pegajosas; etc.; porém não se limitam a eventos fisiológicos, nem são estes as causas do estado ansioso: há também a presença de componentes comportamentais e cognitivos, como relatos de sensações desagradáveis em ocasiões caracterizadas pela impossibilidade de fuga, antecipação da punição e separação do apoio (familiares/amigos próximos). A frequência e a intensidade desses componentes, em contextos específicos, são parâmetros fundamentais na caracterização da ansiedade. A análise do comportamento, no entanto, não se limita à descrição de topografias de reações, sejam estas respondentes ou operantes. O que se busca é a identificação de eventos cruciais na história passada ou presente de um indivíduo, que desencadeiam os componentes respondentes e operantes e geram, frequentemente, alguns subprodutos emocionais e cognitivos. Entre esses eventos cruciais está a exposição ao controle aversivo (punição, ameaça de punição, reforçamento negativo).

De acordo com Holland e Skinner (1961/1974, p. 221), "ansiedade é um nome comum para a predisposição 'emocional' gerada por um estímulo aversivo condicionado. A ansiedade é pois gerada por um estímulo aversivo condicionado". A ansiedade faz parte de um conjunto cada vez mais amplo de estudos sobre estados emocionais conduzidos por analistas do comportamento (p. ex., Banaco, 2001; Coêlho \& Tourinho, 2008; Darwich \& Tourinho, 2005; Dymond

2 No presente texto serão usadas de forma intercambiável as expressões "ansiedade matemática", "ansiedade em relação à matemática", "ansiedade diante da matemática", que são equivalentes à expressão inglesa "mathematics anxiety".
\& Roche, 2009; Friman, Hayes \& Wilson, 1998; Zamignani \& Banaco, 2005). Esses estudos são unânimes em apontar que eventos passados ou presentes determinam as emoções e sentimentos em um indivíduo. Skinner (1971) destaca que não se pode negar a existência de um mundo privado, mas ao mesmo tempo afirma que esse mundo privado compartilha da mesma natureza do mundo exterior, portanto pode ser estudado e é tão importante quanto qualquer outro fenômeno. A posição skinneriana possibilita a investigação dos estados internos e daquilo que comumente tem sido chamado de subjetividade, incluindo a cognição e os sentimentos (Cunha \& Borloti, 2005). Não há, pois, uma posição simplista ou uma desconsideração dos sentimentos e do nosso mundo interno, embora estes não sejam identificados como as causas de nossos comportamentos ${ }^{3}$.

No caso da ansiedade em relação à matemática, esse conjunto de reações gerais, observado na ansiedade crônica, é relatado por indivíduos quando estão, especificamente, diante de situações que exigem a manipulação e aplicação de conhecimentos matemáticos. Desse modo, o ponto crucial reside no fato de um indivíduo relatar desconfortos, semelhantes aos já citados, quando exposto a situações nas quais está presente a matemática.

Como facilmente se pode inferir, a ansiedade em relação à matemática é mais frequentemente identificada entre estudantes, em função da alta probabilidade de se depararem com cálculos e outras relações matemáticas ao longo de sua formação escolar (Baylor, Shen, Warren \& Park, 2004; Tobias, 1978; Toohey, 2002; Turner et al., 2002). Tipicamente, um estudante com ansiedade diante da matemática não conseguirá se concentrar ao fazer exercícios dessa disciplina em sua casa; poderá ficar agressivo ao ser questionado pelo professor na sala de aula sobre alguma atividade, apresentar taquicardia ao realizar um exame de matemática, entre outras reações. As reações fisiológicas que se apresentam nessas ocasiões impedem o aluno de apresentar um bom desempenho nas tarefas que envolvem a matemática, prejudicando-o tanto nas situações em que ocorre a ansiedade quanto em tarefas que deverão ser realizadas posteriormente, como um exame de vestibular, ou até mesmo nas situações vivenciadas no dia a dia (Carmo, 2011).

É fundamental identificar as causas desse padrão típico de reações emocionais à matemática,

Aspectos complementares da definição de ansiedade serão abordados na próxima seção. 
pois possibilitará o desenvolvimento de procedimentos que ajudem os indivíduos a reverter ou, pelo menos, minimizar os efeitos da ansiedade em relação à matemática. Os propósitos do presente trabalho são (a) apresentar resultados de estratégias e procedimentos que têm ajudado indivíduos a superar ou lidar melhor com a ansiedade em relação à matemática, e (b) propor indicações gerais de organização de programas de redução e prevenção da ansiedade em relação à matemática nas escolas. Esses objetivos surgiram a partir da constatação de que no Brasil não há, na literatura acadêmica, relatos de programas desenvolvidos com o propósito de reversão dessa ansiedade específica ${ }^{4}$.

Procedeu-se a um levantamento da literatura nacional e internacional, a partir da consulta às seguintes bases de dados eletrônicas: PsycInfo; Biblioteca Virtual de Saúde (BVS); Web of Science; Science Direct; Periódicos Capes. Procurou-se abranger artigos de periódicos científicos, livros, capítulos de livros, teses e dissertações. Os descritores utilizados foram: math(ematics) anxiety; math(ematics) fear; remediation strategies; reversion of math anxiety; reduction of math anxiety; aversive control. $\mathrm{O}$ levantamento inicial possibilitou a seleção de estudos que apresentavam dados empíricos de redução de ansiedade à matemática, e que partiram da aplicação de técnicas e uso de estratégias psicoterápicas e/ou educacionais. Após a seleção dos textos que atendiam ao critério de relatos de intervenção, foram conduzidas leituras de análise que possibilitaram destacar: (a) concordâncias entre os diversos estudos; (b) discrepâncias nas informações e resultados encontrados; (c) resultados obtidos com as técnicas e estratégias de reversão; (d) indicações e subsídios ao delineamento de um programa de reversão de ansiedade em relação à matemática. $\mathrm{O}$ presente artigo ater-se-á aos itens (c) e (d), que delimitam seus objetivos.

4 No Brasil foram produzidos diversos estudos relevantes acerca de atitudes relacionadas à matemática, alguns indicativos de alterações das atitudes negativas e algumas estratégias de hábitos de estudo, sendo que parte significativa desses estudos foi conduzida por Márcia Regina F. de Brito e seus colaboradores (para exemplificar, indicamos Gonçalez \& Brito, 2001 e Silva, Brito \& Vendramini, 2002). Porém, a falta de programas sistemáticos desenvolvidos e aplicados visando à remediação (reversão), e mesmo à prevenção de ansiedade à matemática, denuncia uma importante carência em nosso meio educacional.

\section{FATORES ENVOLVIDOS NA PRODUÇÃO DE ANSIEDADE EM RELAÇÃO À MATEMÁTICA}

A ansiedade em relação à matemática não é inata nem está diretamente relacionada a transtornos de aprendizagem, como a discalculia, ou a transtornos lesionais, como a acalculia (Ashcraft, 2002; Carmo, 2011). Uma das fontes primárias da ansiedade diante da matemática está na história escolar do indivíduo, na qual se podem identificar experiências negativas marcantes na tentativa de aprender matemática (Carmo, 2011); mas também outros fatores cruciais podem ser identificados. Nossa cultura ocidental divulga informações controversas em relação à matemática, relacionando-a a algo de difícil apreensão, somente acessível a poucos indivíduos, que exige muito esforço e dedicação para ser dominado. Em casa é comum que os familiares divulguem essas regras às crianças. $\mathrm{Na}$ escola, muitos professores reforçam tais informações, seja por meio de regras inadequadas passadas aos alunos (matemática é difícil; só existe uma solução para cada problema; só o professor pode dizer se a solução está certa ou não), seja por meio de metodologias de ensino inadequadas seja pelo uso de controle aversivo.

Assim, o estudo da ansiedade matemática deve levar em conta não apenas aspectos descritivos das reações respondentes e operantes apresentadas pelo indivíduo, uma vez que estas auxiliam apenas na identificação de padrões comportamentais típicos, os quais, embora relevantes e imprescindíveis, não abrangem a totalidade do fenômeno e não são suficientes para o entendimento do indivíduo com ansiedade matemática e seu contexto de vida. Faz-se necessário levar em conta que os comportamentos são determinados por três diferentes histórias em interação: filogênese, ontogênese e cultura (Skinner, 1981). Mais uma vez, a ênfase deve ser dada à história passada do indivíduo e a aspectos atuais que podem fragilizar o indivíduo diante de situações relacionadas à matemática. Nesse sentido, há componentes diretamente relacionados à história de aprendizagem escolar, e há componentes que estão enraizados no modo como esse indivíduo aprendeu a lidar com eventos ansiogênicos ou estressantes. Em função da limitação de espaço, a ênfase do presente estará centrada nos fatores relacionados ao contexto de aprendizagem escolar, particularmente os componentes aversivos, regras e metodologias inadequadas.

Skinner (1968), ao tratar do ensino escolar, identificou que uma estratégia comumente utilizada por professores é o controle aversivo (também chamado de controle coercitivo), que consiste em um 
conjunto de operações que tem por efeito a diminuição da frequência de determinados comportamentos. Essas operações são: (a) apresentação de estímulo aversivo contingente a uma dada resposta do aluno, ou seja, punição; (b) retirada de estímulo reforçador contingente a uma determinada resposta do aluno, isto é, reforçamento negativo; (c) ameaça de punição, ou seja, anunciar (verbalmente ou por meio de outras sinalizações) que uma contingência aversiva poderá ocorrer.

A utilização do controle aversivo pode gerar, e frequentemente gera, subprodutos indesejáveis no repertório do aluno, o qual passa a apresentar respostas de fuga e esquiva diante de situações nas quais foi punido anteriormente (Mazzo \& Gongora, 2007). Como exemplo, temos supressão condicionada (parar de responder), os subprodutos emocionais (como o medo e a ansiedade) e o aumento de respostas de agressão quando uma pessoa está sob controle aversivo. A ansiedade consiste em um conjunto de respostas reflexas eliciadas por um estímulo aversivo que interfere em ações operantes, suprimindo operantes reforçados positivamente e facilitando operantes negativamente reforçados (Skinner, 1968). Quando a ansiedade está presente, notam-se reações desfavoráveis para o indivíduo e incontroláveis a tal ponto que interferem em padrões fisiológicos importantes para a economia do organismo (alterações na pressão arterial e na frequência cardíaca, por exemplo). O aparecimento da ansiedade está diretamente relacionado à utilização do controle aversivo, particularmente quando estão presentes determinadas características da estimulação aversiva, como: severidade (intensidade) da estimulação aversiva; ocorrência amiúde (alta frequência) de estimulação aversiva; forma com que a estimulação aversiva é aplicada; imediaticidade, isto é, a estimulação aversiva contingente e contígua ao comportamento a ser suprimido; incontrolabilidade, ou seja, a total incapacidade de evitar ou fugir da estimulação aversiva (Fantino, 1973; Mazzo \& Gongora, 2007).

No caso da aprendizagem da matemática escolar, erros e mau desempenho ao fazer um exercício de matemática ou ao responder incorretamente a uma pergunta do professor podem ter como consequência uma das formas de controle aversivo. Caso esse controle apresente as características apontadas anteriormente, é bastante provável que haja um aumento na ocorrência de erros, o que gera mais estimulação aversiva. Esta é, presumivelmente, a base da ansiedade à matemática. Por outro lado, é importante ressaltar que a utilização de controle aversivo é mantida pela eficácia dessa técnica (Hubner, 1998; Sidman, 1989). Ao punir o mau desempenho de um aluno, o professor obtém, na maioria das vezes, uma resposta de atenção do aluno ao erro apontado. O aluno pode reforçar o uso do controle aversivo pelo professor ao melhorar imediatamente seu desempenho, mesmo que nesses casos a melhora seja apenas aparente e mantida por esquiva. Em outras palavras, o aluno passa a estudar para se esquivar à punição e uma eventual melhora no desempenho é passageira, limitando-se ao momento da prova (Sidman, 1989). Nesse caso, há estudantes que conseguem boas pontuações, mas não conseguem ultrapassar um nível meramente mecânico de resolução dos exercícios (Fiorentini, 1995).

Embora seja fundamental identificar o uso do controle coercitivo em contextos de ensino e aprendizagem da matemática, torna-se importante ressaltar que ansiedade não deve ser reduzida a aversão à matemática, uma vez que essa aversão não é um subproduto gerado apenas pelo controle coercitivo. Para além do uso de punição e ameaça de punição, a ansiedade em relação à matemática tem como um dos principais fatores geradores a maneira como essa disciplina é ensinada na escola (Carmo, Cunha \& Araújo, 2008; Newstead, 1998). Os professores passam a matéria de maneira enfadonha, não estimulam debates sobre a matemática e seus conteúdos diversificados reforçam respostas que são dadas com exatidão, sem levar em conta o raciocínio desenvolvido, cobram rapidez na resolução dos exercícios e exigem memorização de regras para esse fim. Resumidamente, muitos professores não incentivam nem estabelecem condições para o "pensar matematicamente", isto é, para o desenvolvimento de estratégias de resolução de problemas e de análise de relações matemáticas presentes em situações cotidianas e em situações escolares.

Outro aspecto que tem recebido pouca atenção em nosso país é a possibilidade de muitos professores das séries iniciais do Ensino Fundamental também apresentarem ansiedade em relação à matemática ou, pelo menos, aversão e dificuldades de entendimento de fatos elementares da matemática (Utsumi \& Lima, 2008). Parte significativa dos professores que atuam nas séries iniciais é formada em Pedagogia e não recebe, durante a graduação, a oportunidade de uma formação mais sólida quanto ao conhecimento e a metodologias de ensino da matemática. $\mathrm{O}$ contato com a área de investigação e aplicação chamada de Educação Matemática é mínimo. Além disso, muitos professores que ensinam matemática no primeiro ciclo do Ensino Fundamental possuem uma história de 
fracasso em matemática e manifestaram interesse por uma disciplina científica das chamadas Ciências Humanas, para evitar um contato mais próximo e decisivo com a matemática. Todos esses fatores podem concorrer para um baixo aproveitamento tanto dos professores quanto dos alunos nas aulas de matemática das séries iniciais, conforme pode ser visto nos estudos de Baumann, 2009; Utsumi \& Lima, 2008. Por outro lado, alguns estudos (Beilock, Gunderson, Ramirez, \& Levine., 2010; Peker, 2009) apontam que professoras com ansiedade em relação à matemática podem oferecer às alunas modelos e regras inadequados, aumentando o preconceito amplamente difundido de que meninos são melhores que meninas em matemática.

A partir dessas observações, pode-se afirmar que a ansiedade em relação à matemática não é simples de ser abordada, por se tratar de um fenômeno multideterminado. Conforme apontado anteriormente, deve-se considerar a educação tanto em casa como na escola, a influência parental sobre os filhos, como eles lidam com a matemática e como a classificam. A própria sociedade apresenta a matemática como algo de difícil apreensão, como se apenas os alunos mais inteligentes (o que quer que isso signifique) conseguissem ser bem-sucedidos na resolução dos cálculos e exercícios. Isso gera sentimentos de incapacidade em alguns alunos e pensamentos do tipo "nunca vou conseguir tirar uma boa nota nessa disciplina, pois não sou suficientemente inteligente para isso", "não me encaixo no perfil exigido pela disciplina", entre outros. Estas declarações dos alunos podem se manifestar de forma explícita ou encoberta. São regras e autorregras que se caracterizam, tipicamente, por serem autoatribuições negativas, o que está diretamente relacionado à autoestima e autoavaliação em relação ao seu desempenho em matemática. Desse modo, controle coercitivo, metodologias de ensino inadequadas, formação básica e continuada insuficiente dos professores e fatores culturais e familiares concorrem para gerar o quadro chamado de ansiedade em relação à matemática, o qual poderia, resumidamente, ser descrito como padrões de fuga e esquiva diante de situações que envolvem a matemática, acompanhado por reações fisiológicas desagradáveis e reações cognitivas autodepreciativas (regras e autorregras).

A identificação dos fatores geradores de ansiedade diante da matemática possibilitou a condução de estudos e a proposição de estratégias para auxiliar estudantes com dificuldades extremas em matemática que, notadamente, poderiam ser enquadrados como casos típicos de ansiedade em relação à matemática. A seguir serão expostos alguns desses estudos e suas contribuições para a proposição de programas de reversão e prevenção da ansiedade em relação à matemática.

\section{ESTRATÉGIAS E PROCEDIMENTOS DE REVERSÃO ${ }^{5}$ DA ANSIEDADE EM RELAÇÃO À MATEMÁTICA}

Em levantamentos acerca das estratégias de reversão de ansiedade à matemática (Carmo, 2011; Carmo, Cunha \& Araújo, 2008) foram identificados três grandes focos passíveis de intervenção: a família, a escola e o estudante. Embora todas as estratégias visem auxiliar o indivíduo, é fundamental ficar claro que este responde a contingências presentes em diferentes ambientes que o afetam diretamente, daí a necessidade de auxiliar professores e familiares nesse processo. De modo geral, os levantamentos feitos por Carmo (2011) e Carmo, Cunha e Araújo (2008) indicaram que na literatura são encontrados relatos de utilização de procedimentos clínicos, particularmente dos modelos de terapia comportamental e cognitivocomportamental. Além disso, também são relatados estudos envolvendo procedimentos em sala de aula, comparação de metodologias de ensino, e presença de monitores.

Estudos que implementaram mudanças no ambiente de estudo e nas estratégias de ensino indicam um ganho na aprendizagem e na redução de estresse em estudantes da escola elementar (Iossi, 2007; Perry, 2004; Rossnan, 2006; Toumasis, 2004; Wei, 2010), da escola secundária (Hellum-Alexander, 2010; Meece, Wigfield \& Eccles, 1990) e universitários/adultos (Iossi, 2007; Latiolais \& Laurence, 2009). As estratégias envolveram principalmente rearranjos no ambiente de estudo, tais como presença de monitores, trabalhos em pequenos grupos, acompanhamento individualizado, rodas de conversa sobre matemática, procedimentos de ensino individualizado e ensino a distância via computador. As descrições de resultados, a seguir, poderão fornecer indicativos relevantes à proposição de programas de reversão e prevenção de ansiedade em relação à matemática, e serão retomados na última seção do presente artigo.

Tobias (1978) aponta comportamentos que devem ser evitados pelos professores em sala de aula. Entre eles estão: pressionar os alunos para responder rápida

O termo "reversão" tem sido usado na literatura que trata de estratégias de auxílio a indivíduos com ansiedade à matemática. 
e corretamente as questões propostas sobre a matéria; estimular competição entre colegas; aplicar muitos testes e humilhar os alunos que apresentarem algum tipo de dúvida sobre matemática ou demorarem a entender o raciocínio exigido em determinado exercício.

Hendel e Davis (1978) descreveram de que maneira as atividades de programas educacionais e as estratégias de intervenção/aconselhamento devem ser usadas para reduzir a ansiedade em relação à matemática, particularmente em mulheres que voltaram para a escola quando adultas. O estudo contou com 69 mulheres que responderam à escala MARS (Mathematics Anxiety Rating Scale) antes e após a aplicação das estratégias de intervenção/aconselhamento. A escala MARS contém 94 itens com escore variando de 1 a 5, aos quais correspondem intensidades crescentes de ansiedade. Depois de responderem à MARS, as mulheres participavam de um diagnóstico clínico para medir as habilidades matemáticas e saber como a ansiedade diante da matemática está afetando o desempenho delas na disciplina. Além disso, elas poderiam frequentar cursos especiais de matemática, os quais continham instrutores capazes de sensibilizar as alunas com dificuldade, tinham direito a pedir auxílio a um grupo de suporte que aplicava exercícios para reduzir a ansiedade em relação à matemática e também poderiam participar de um fórum a fim de discutir experiências prévias com a disciplina. Os resultados apontaram que foi mais efetiva a redução de ansiedade em alunas matriculadas nos cursos de matemática e que pediram auxílio ao grupo de suporte. As mulheres que utilizaram as três opções oferecidas pela pesquisa obtiveram decréscimo de 75 pontos nos níveis de ansiedade em relação à matemática, enquanto as que fizeram apenas o diagnóstico clínico reduziram em apenas 14 pontos seus níveis de ansiedade.

Turner et al. (2002) investigaram a relação entre o ambiente de aprendizagem e o relato de estudantes sobre estratégias para se evitar a matemática. Participaram do estudo 1092 estudantes da sexta série (equivalente ao nosso Ensino Fundamental) (52\% mulheres / $48 \%$ homens $-70 \%$ euro-americanos $/ 30 \%$ afro-americanos) e nove professores, em nove escolas diferentes. No procedimento foi utilizada uma escala de tipo Likert. A escala possibilitava medir o comportamento do aluno de evitar procurar por ajuda, e variava de 1 a 5 (de não verdadeiro até muito verdadeiro). Além do comportamento de esquiva, também foram medidos os comportamentos de autossabotagem, percepção do aluno sobre o mestre e percepção dos objetivos e da estrutura da escola. Os resultados indicaram que $13 \%$ dos estudantes assinalaram utilizar a autossabotagem enquanto estudam, 9\% evitam pedir ajuda aos colegas, professores ou parentes e $10 \%$ evitam novidade no que se refere a um conteúdo novo da disciplina; o restante não assinalou nenhuma das alternativas.

Os resultados também apontam uma relação entre o tipo de discurso do professor e o uso de estratégias de esquiva pelos estudantes. Em seu estudo, Turner et al. (2002) identificaram três tipos de discurso capazes de influenciar o desempenho dos alunos: discurso instrucional, discurso motivacional e discurso que induz à organização de ideias. O discurso instrucional transmite significados (esclarecer conceitos, palavraschave) e transfere responsabilidade; o discurso motivacional tem a função de expressar emoções positivas, focar no aprendizado e incentivar ajuda/colaboração entre os colegas; e o discurso que induz à organização de ideias fornece direção aos estudos e responde a questões sobre procedimento dos exercícios. Dentre os professores participantes do estudo, $52 \%$ a $68 \%$ apresentaram discurso instrucional e de $20 \%$ a $30 \%$ apresentaram o discurso que leva à organização de idéias; no entanto, segundo Turner e colaboradores, em alguns casos os docentes utilizavam de maneira desestruturada e pouco sistemática os três tipos de discurso.

O tipo de discurso do professor parece ser um aspecto primordial na identificação e intervenção em casos de ansiedade em relação à matemática, e os autores levantaram a seguinte questão: como podemos melhorar o discurso do professor para que o aluno se sinta interessado na disciplina? Turner e seus colegas observaram o discurso de alguns professores muito bem-sucedidos no ensino da matemática a fim de sanar (em partes) sua dúvida. Eles concluíram que no discurso motivacional aparecem brincadeiras e piadas sobre a matemática que deixam os alunos envolvidos nas aulas. Além disso, algumas frases ditas com frequência pelos alunos, como: "Eu não sei fazer isso" são rapidamente rebatidas pelo professor que apresenta um bom discurso motivacional, cuja resposta seria: "Se eu sei fazer, é claro que você também sabe". Caso algum aluno não entenda a explicação ou esteja com dificuldades insistentes nos exercícios, a matéria é explicada passo a passo, novamente, se for preciso.

Os resultados obtidos por Turner e colaboradores foram corroborados por Baylor, Shen e Warren (2005), os quais indicam que agentes educacionais motivadores oferecem um excelente modelo de suporte a estudantes com ansiedade em relação à matemática. No estudo de Baylor e colaboradores, 67 
estudantes que apresentavam dificuldades em matemática e ansiedade em relação à matemática foram expostos a problemas de percentagem e interagiam com um dentre quatro modelos de agentes educacionais criados graficamente: agentes que motivavam; agentes que não motivavam; agentes que expressavam afetividade; agentes que não expressavam afetividade. Os estudantes expostos ao modelo motivador apresentaram melhora no desempenho matemático quando comparados aos estudantes expostos aos outros modelos.

Hutter (1995) objetivou identificar quais efeitos teria sobre a ansiedade em relação à matemática a exposição às seguintes situações e, se os tivessem, quais seriam: (a) o número crescente de problemas escritos (sentenças) que eram apresentados pelo menos uma vez a cada dia; (b) duas vezes por semana escrever sobre seus próprios sentimentos e dificuldades no momento em que tentam resolver exercícios de matemática; (c) trabalhos em grupo pelo menos uma vez por semana; (d) emissão de elogios contingentes ao desempenho, diversas vezes por dia. Participaram 47 estudantes de ensino médio, classificados em dois grupos, quanto ao desempenho em matemática: 21 estudantes abaixo da média e 26 acima da média. Ambos os grupos foram expostos às quatro situações durante seis semanas. Além dessas situações, participaram de treino de inoculação de estresse e dessensibilização sistemática, e aplicação da escala MARS no início e no final da intervenção.

Os resultados apontaram que não houve decréscimo na pontuação da escala MARS para os estudantes abaixo da média, enquanto os estudantes acima da média apresentaram ligeira diminuição na pontuação. Apesar de não terem sido identificadas alterações significativas, Hutter (1995) propõe que os grupos de estudo ajudam na difusão da responsabilidade entre alunos. Escrever sobre seus próprios sentimentos quando encontram dificuldades nos exercícios de matemática pode funcionar como uma dica acerca dos obstáculos gerados pelos próprios estudantes. Em outras palavras, no caso específico, escrever sobre seus próprios sentimentos deixava os estudantes sensíveis às próprias dificuldades e os ajudava a identificar quando a ansiedade iniciava e dessa forma conseguiam diminuí-la sem interromper o estudo. Esse processo foi chamado por Tobias (1978) de "autocura". Por fim, Hutter propôs que enxergar a matemática como significativa e relevante traz motivação para o estudo dessa matéria e os elogios aumentam a perseverança dos alunos na resolução do exercício.
Colombini, Shoji e Pergher (2012) relatam um estudo de remediação de hábitos de estudos inadequados em um estudante adolescente que, provavelmente, apresentava ansiedade em relação à matemática. Os relatos verbais do adolescente enfatizavam aversão total à matemática. $\mathrm{O}$ estudo de caso conduzido envolveu a intervenção de um acompanhante terapêutico, que desenvolveu alguns procedimentos de reorganização dos hábitos de estudos (uso da agenda, horários, organização de material, aproximação sucessiva à matemática, reforço pós-estudo). A intervenção resultou em ganhos na qualidade e organização dos hábitos de estudo, aproximação à matemática e aumento nas notas. Também houve diminuição das verbalizações negativas relacionadas à matemática.

Quanto às estratégias de terapia, a técnica mais difundida e estudada é a dessensibilização sistemática, desenvolvida e aplicada no âmbito da clínica comportamental com vista a estabelecer o contracondicionamento em situações que eliciam respostas emocionais negativas (McGlynn, Smitherman, \& Gothard, 2004; Richardson \& Suinn, 1973; Schneider \& Nevid, 1993). McGlynn, Smitherman e Gothard (2004), realizaram um amplo levantamento da literatura acerca das aplicações e benefícios da dessensibilização sistemática e concluíram que, apesar de um ligeiro declínio no número de publicações sobre a técnica, esta ainda é amplamente utilizada e recomendada por terapeutas.

Alguns estudos compararam os efeitos da dessensibilização sistemática, tradicional ou com variações, com o efeito de outras técnicas terapêuticas, ou ainda o efeito da associação da dessensibilização com outras técnicas. Schneider e Nevid (1993), por exemplo, investigaram os efeitos isolados de inoculação de estresse e dessensibilização no tratamento de ansiedade em relação à matemática em 39 universitários. Os resultados mostraram que ambas as técnicas foram igualmente eficazes na redução da ansiedade em situações de estudo da matemática. Richardson e Suinn (1973) compararam o efeito da exposição à dessensibilização sistemática tradicional e a uma variante da dessensibilização, chamada de dessensibilização massiva acelerada, em estudantes universitários com alto grau de ansiedade em relação à matemática. A diferença básica entre as modalidades de dessensibilização era que na forma tradicional os participantes eram expostos a todos os itens da hierarquia de situações ansiogênicas, enquanto na outra modalidade os participantes eram expostos somente aos três itens mais ansiogênicos da hierarquia. Os resultados foram positivos para ambos 
os grupos e não apresentaram diferenças estatisticamente significativas.

\section{IMPLEMENTAÇÃO DE PROGRAMAS DE PREVENÇÃO E REDUÇÃO DA ANSIEDADE EM RELAÇÃO À MATEMÁTICA: ALGUNS INDICATIVOS}

Os resultados de pesquisas relatados na seção anterior, embora escassos, oferecem indicativos importantes para a formulação de programas que visem auxiliar estudantes com ansiedade em relação à matemática, bem como prevenir o surgimento de novos casos.

Uma proposta desenvolvida por Toohey (2002) será aqui apresentada como exemplo de um programa bem-sucedido aplicado a 30 estudantes do ensino secundário claramente identificados como tendo ansiedade diante da matemática. Toohey chamou de "plano de ação para auxiliar estudantes com ansiedade em relação à matemática", o qual, resumidamente, foi desenvolvido na seguinte ordem:

1) identificação de casos de ansiedade em relação à matemática na escola, por meio de observação direta, observação do desempenho, aplicação de questionários de ansiedade em relação à matemática (escalas);

2) mudança do ambiente em sala de aula, relacionadas à metodologia de ensino e postura do professor, como: uso frequente e explícito de mensagens positivas (motivadoras); regras, objetivos e expectativas claramente anunciados; atmosfera positiva (evitar frases e ações depreciativas); o professor só convidar o estudante a ir ao quadro se este explicitamente levantar a mão (como indicativo de que está à vontade); explicações claras e detalhadas das tarefas de casa; respostas verbais e resoluções erradas devem ser imediatamente consequenciadas com possibilidade de novas tentativas e, se for o caso, indicação imediata da resposta ou resolução correta, acompanhada de explicação; ausência de tempo limite para as avaliações; etc.

3) Intervenção com os estudantes: entrevistas individuais a fim de estabelecer segurança nos estudantes e assegurar que o professor está de acordo em alterar sua metodologia de ensino e comportamentos em sala de aula; uso de monitores para auxílio individualizado aos estudantes.

Como indicações gerais, estudos em Análise do Comportamento podem também contribuir para que o ambiente de sala de aula promova uma aprendizagem mais eficaz e livre de erros. Carmo (2010) apresenta os seguintes indicativos que ajudam o professor a rever sua dinâmica de trabalho em sala de aula: 1) utilizar a avaliação qualitativa ao longo das aulas; 2) redimensionar a noção de erro e seu tratamento; 3) criar oportunidades de acerto; 4) revisar periodicamente os conteúdos ensinados; 5) dividir o conteúdo em unidades menores; 6) proporcionar aos alunos abertura para expor suas dúvidas e dificuldades; 7) atentar para os comandos dados nas questões da prova; 8) diversificar os instrumentos de avaliação; 9) verificar se alguns alunos apresentam dificuldades ou limitações específicas e precisam de uma prova diferenciada, com o auxílio de um acompanhante ou monitor, e um tempo a mais para responder; e 1) aproveitar didaticamente o momento de devolução da prova.

Carmo explicita melhor alguns desses itens, como segue:

- Utilizar a avaliação qualitativa ao longo das aulas. Um professor atento estará sensível a alguns comportamentos gerais que poderão ser indicativos importantes, como, por exemplo: (a) os alunos frequentemente não conseguem atingir os objetivos propostos: neste caso é possível que o conteúdo precise ser revisto, dividido em unidades menores ou seus pré-requisitos precisavam ser garantidos; (b) os alunos não conseguem realizar as atividades propostas: pode ser que essas atividades não sejam adequadas e seja necessário diminuir a complexidade das mesmas ou, ainda, pode ocorrer que as atividades propostas exijam um desempenho que não foi ensinado antes; (c) silêncios, não engajamento na dinâmica da aula, dispersão de atenção: muitas vezes uma simples conversa pode esclarecer a respeito do que precisa ser alterado no planejamento ou na dinâmica de trabalho.

- Redimensionar a noção de erro e seu tratamento: liberar consequências aversivas referentes aos erros cometidos poderá gerar baixa autoestima nos estudantes. Alunos que comentem erros frequentes são vistos como alunos que têm dificuldades de aprendizagem; porém essa visão é bastante equivocada e pode desvirtuar o trabalho do professor. Erros frequentes geram fracasso na aprendizagem e o aluno passa a evitar ou fugir do contato com a disciplina. Cabe ao professor verificar que alternativas metodológicas possibilitam diminuir a ocorrência de erros e aumentam as chances de sucesso do aprendiz. Erros são vistos como um desempenho que não 
atingiu um critério arbitrariamente definido pelo professor. Cabe, então, ao professor verificar se os critérios de desempenho adotados não estão acima da capacidade dos alunos. Erros não devem ser vistos como um desempenho apenas do aluno, e sim, como uma produção coletiva, pois só há "erros" em função de que houve a aplicação de um procedimento quel pode não ter garantido ao aluno os requisitos necessários à aprendizagem. Erros não devem servir de recurso classificatório, e sim, de indicativo de que o planejamento precisa ser revisto.

- Criar oportunidades de acertos: experimentar o sucesso na aprendizagem é fundamental para manter o aluno motivado nos estudos.

- Atentar aos comandos dados nas questões de prova: elaborar comandos simples, diretos e que estabeleçam claramente o que se quer

- Aproveitar didaticamente o momento de devolução da prova didaticamente: não simplesmente anunciar a nota, mas também comentar as questões, identificar os erros mais comuns (sem dizer quem os cometeu) a fim de dirimir dúvidas.

Afora esses indicativos dados até aqui, faz-se necessário lembrar que um programa desse porte deveria envolver toda a escola, se não todo o sistema de ensino, uma vez que não pode ser assumido por alguns agentes isoladamente. Por outro lado, como o foco maior está na mudança de comportamentos do estudante, esta só poderá se efetivar a partir da alteração de contingências na escola e na família e a partir da implementação de suporte externo, seja este psicoterápico, motivacional ou metodológico.

As soluções podem ser diversas, mas a grande questão é como e onde começar a intervenção psicológica para caminharmos rumo à superação de tal problema. Seria melhor a implantação de um programa de intervenção na escola ou em casa? Quiçá um trabalho em conjunto seria mais vantajoso? A segunda opção parece chegar mais perto da realidade. A intervenção feita separadamente, primeiro na escola e depois em casa, ou ao contrário, começaria incompleta, pois a instituição escolar precisa do apoio familiar nessa questão e a família precisa dos professores e psicólogos fornecidos pela escola.

A implantação de programas de ensino em que esteja presente o reforço positivo é algo de extrema importância a ser realizado na escola. Isso requer dedicação dos professores e incentivo tanto financeiro quanto motivacional oferecido pelo governo, no caso de escolas públicas. São necessárias atitudes governamentais como o fornecimento de cursos sobre como utilizar técnicas comportamentais que possam ser bem-sucedidas no ensino da matemática, a contratação de psicólogos que possam auxiliar os docentes nessa tarefa e a realização de intervenções individualizadas com alunos que apresentem de alta a extrema ansiedade em relação à matemática.

Nas escolas particulares, pode-se trabalhar com o oferecimento de mais plantões de matemática com a participação de professores e psicólogos nestas ocasiões. Além disso, atitudes da coordenação como as já citadas anteriormente podem ter consequências benéficas para os alunos ao longo do tempo.

A atenção dos pais/familiares na questão da ansiedade em relação à matemática deve ser destacada como necessidade básica pela coordenação e/ou professores durante as reuniões nas quais ocorrem entrega de boletins ou são dadas informações adicionais sobre o comportamento dos alunos na escola. O apoio familiar dado de maneira orientada (tanto por professores como por psicólogos) poderá ser a mola propulsora para um bom desempenho nas atividades escolares. Os pais que estiverem na zona de conforto, na qual eles enxergam a escola como a grande responsável por solucionar todas as dificuldades apresentadas pelos alunos, poderiam ser chamados em particular pela coordenação para uma conversa e, a partir desta, ser-lhes esclarecida toda a nova política educacional e a tentativa de implantação de programas de intervenção para alunos com ansiedade diante da matemática.

No ambiente sala de aula, a utilização de brincadeiras e dinâmicas que exigem a participação dos alunos durante as aulas de matemática podem servir para transformar essa disciplina em algo mais "palpável" para os alunos. O caráter abstrato da disciplina é considerado um dos motivos para o baixo desempenho dos estudantes com dificuldades em matemática.

Enfim, novas pesquisas são necessárias, mas podemos afirmar que já temos condições de esboçar programas de auxílio específico a estudantes com ansiedade em relação à matemática. A oferta de serviços desta natureza é uma necessidade premente em nosso país e poderá contribuir não apenas para a reversão, mas também para a prevenção de ansiedade em relação à matemática.

\section{REFERÊNCIAS}

Ashcraft, M. H. (2002). Math anxiety: Personal, educational, and cognitive consequences. Current Directions in Psychological Science, 11(5), 181-185. 
Banaco, R. (2001). Alternativas não aversivas para tratamento de problemas de ansiedade. In M. L. Marinho \& V. E. Caballo (Orgs.), Psicologia clínica e da saúde (pp. 197212). Londrina, PR: Eduel.

Baumann, A. P. P. (2009). Características da formação de professores de matemática nos anos inicias do ensino fundamental com foco nos curso de pedagogia e matemática. Dissertação de Mestrado não publicada, Programa de Pós-Graduação em Educação Matemática, Universidade Estadual Paulista, Rio Claro, SP.

Baylor, A. L., Shen, E., Warren D., \& Park, S. Supporting learners with math anxiety: The impact of pedagogical agent emotional and motivational support. Anais da Conference on Intelligent Tutoring Systems, Maceió, Brasil, 2004.

Beilock, S. L., Gunderson, E. A., Ramirez, G., \& Levine, S. C. (2010). Female teachers' math anxiety affects girls' math achievement. Proceedings of the National Academy of Sciences of the United States of America, 107(5), 18601863.

Carmo (2010). Fundamentos psicológicos da educação. Curitiba: Ibpex.

Carmo, J. S. (2011). Ansiedade à matemática: identificação, descrição operacional e estratégias de intervenção. In F. Capovilla (Org.), Transtornos de aprendizagem: progressos em avaliação e intervenção preventiva e remediativa (pp. 249-255). São Paulo: Memnon.

Carmo, J. S., Cunha, L. O., \& Araújo, P. V. S. (2008). Analise comportamental da ansiedade à matemática: conceituação e estratégias de intervenção. In W. C. M. P. Silva (Org.), Sobre comportamento e cognição: análise comportamental aplicada (pp. 185-195). Santo André, SP: ESETec.

Coêlho, N. L., \& Tourinho, E. Z. (2008). O conceito de ansiedade na análise do comportamento. Psicologia: Reflexão e Crítica, 21(2), 171-178.

Colombini, F., Shoji, F. T., \& Pergher, N. K. (2012). Ansiedade matemática e desenvolvimento de hábitos de estudo: Algumas possibilidades de atuação do acompanhante terapêutico. In C. V. V. B. Pessoa, C. E. Costa \& M. F. Benvenuti (Orgs.) Comportamento em Foco (pp. 131-142). São Paulo: ABPMC.

Cunha, L. S., \& Borloti, E. B. (2005). Skinner, o sentimento e o sentido. In E. B. Borloti, S. R. F. Enumo, \& M. L. P. Ribeiro (Orgs.), Análise do comportamento: Teorias $e$ práticas (pp. 47-57). Santo André, SP: ESETec.

Darwich, R. A., \& Tourinho, E. Z. (2005). Respostas emocionais à luz do modo causal de seleção por consequências. Revista Brasileira de Terapia Comportamental e Cognitiva, 7(1), 107-118.

Dreger, R. M., \& Aiken Jr., L. R. (1957). The identification of number anxiety in a college population. Journal of Educational Psychology, 48(6), 344-351.

Dymond, S., \& Roche, B. (2009). A contemporary behavior analysis of anxiety and avoidance. The Behavior Analyst, $32,7-27$.

Fantino, E. (1973). Aversive control. In J. A. Nevin, \& J. S. Reynolds (Orgs.), The study of behavior: Learning, motivation, emotion, and instinct (pp. 239-279). Glenview, Il: Scott, Foresman and Company.
Fiorentini, D. (1995). Alguns modos de ver e conceber o ensino de matemática no Brasil. Zetetiké, 4, 1-37.

Friman, P. C., Hayes, S. C., \& Wilson, K. G. (1998). Why behavior analysts should study emotion: The example of anxiety. Journal of Applied Behavior Analysis, 31, 137156.

Gonçalez, M. H. C. C., \& Brito, M. R. F. (2001). A aprendizagem de atitudes positivas em relação à matemática. In M. R. F. Brito (Org.), Psicologia da educação matemática: Teoria e Pesquisa (pp. 221-233). Florianópolis: Insular.

Helleum-Alexander, A. (2010). Effective teaching strategies for alleviating math anxiety and increasing self-efficacy in secondary students. Dissertação de Mestrado não publicada, Master in Teaching Thesis, The Evergreen State College.

Hendel, D. D., Davis, S. O. (1978). Effectiveness of an intervention strategy for reducing mathematics anxiety. Journal of Counseling Psychology, 25(5), 429-434.

Holland, J. G., \& Skinner, B. F. (1974). A análise do comportamento. (R. Azzi, Trad.). São Paulo: EPU. (trabalho originalmente publicado em 1961).

Hubner, M. M. C. (1998). Analisando a relação professoraluno: Do planejamento à sala de aula. São Paulo: CLRBalieiros.

Hutter, M. S. (1995). A study to determine the effectiveness of various factors in the reduction of mathematics anxiety. Dissertação de Mestrado não publicada, Programa de PósGraduação em Artes, Rowan University, Glasboro, Nova Jérsei.

Iossi, L. (2007). Strategies for reducing math anxiety in postsecondary students. In S. M. Nielsen, M. S. Plakhotnik (Orgs.), Anais da Sixth Annual College of Education Research Conference: Urban and International Education Section (pp. 30-35). Miami: Florida International University.

Latiolais, M. P., \& Laurence, W. (2009). Engaging mathavoindant college students. Numeracy, 2(1), 1-10.

Mazzo, I. M. B., \& Gongora, M. A. N. (2007). Controle aversivo do comportamento: das definições operacionais aos subprodutos indesejáveis e desejáveis. In W. C. M. P. Silva (Org.), Sobre comportamento e cognição: reflexões teórico-conceituais e implicações para pesquisa (pp. 42-62). Santo André, SP: ESETec.

McGlynn, F. D., Smithermann, T. A., \& Gothard, K. D. (2004). Comments on the status of systematic desensitization. Behavior Modification, 28(2), 194-205.

Meece, J. L., Wigfield, A., \& Eccles, J. S. (1990). Predictors of math anxiety and its influence on young adolescents' course enrollment intentions and performance in mathematics. Journal of Educational Psychology, 82(1), 60-70.

Newstead, K. (1998). Aspects of children's mathematics anxiety. Educational Studies in Mathematics, 36(1), 53-71.

Peker, M. (2009). Pre-service teachers' teaching anxiety about mathematics and their learning styles. Eurasia Journal of Mathematics, Science, \& Techonolgy Education, 5(4), 335345. 
Perry, A. B. (2004). Decreasing math anxiety in college students. College Student Journal, 38(2), 321-324.

Richardson, F. C., Suinn, R. M. (1973). A comparison of traditional systematic desensitization, accelerated massed desensitization, and anxiety management training in the treatment of mathematics anxiety. Behavior Therapy, 4(2), 212-218.

Rossnan, S. (2006). Overcoming math anxiety. Mathitudes, 1, 01-04.

Schneider, W. J., \& Nevid, J. S. (1993). Overcoming math anxiety: A comparison of stress inoculation training and systematic desensitization. Journal of College Student Development, 34, 283-288.

Sidman, M. (1989). Coertion and its fallout. Boston: Authors Cooperative.

Silva, C. B., Brito, M. R. F., \& Vendramini, C. M. M. (2002). Atitudes em relação à estatística e à matemática. PsicoUSF, 7 (2), 89-100.

Skinner, B. F. (1968). The technology of teaching. New York: Appleton-Century- Crofts.

Skinner, B. F. (1971). Beyond freedom and dignity. New York: Alfred A. Knopf.

Skinner, B. F. (1981). Selection by consequences. Science, 213(4507), 501-504.

Tobias, S. (1978). Overcoming math anxiety. New York, NY: Norton.

Toohey, C. W. (2002). An action plan to help students with math anxiety. Dissertação de Mestrado não publicada, Universidade da Pensilvânia.
Toumasis, C. (2004). Cooperative study teams in mathematics classrooms. International Journal of Mathematical Education in Science and Technology, 35(5), 669-679.

Turner, J. C., Midgley, C., Meyer, D. K., Gheen, M., Anderman, E. M., Yongjin, K., \& Patrick, H. (2002). The classroom environment and students' reports of avoidance strategies in mathematics: A multimethod study. Journal of Educational Psychology, 94(1), 88-106.

Utsumi, M. C., \& Lima, R. C. P. (2008). Um estudo sobre as atitudes de alunas de pedagogia em relação à matemática. Educação Matemática em Revista, 24(13), 46-54.

Wei, Q. (2010). The effects of pedagogical agents on mathematics anxiety and mathematics learning. Tese de Doutorado não publicada, Doctor o $f$ Philosophy in Instructional Technology and Learning Sciences Thesis. Utah State University.

Zamignani, D. R., \& Banaco, R. A. (2005). Um panorama analítico-comportamental sobre os transtornos de ansiedade. Revista Brasileira de Terapia Comportamental e Cognitiva, 7, 77-92.

\section{Endereço para correspondência:}

João dos Santos Carmo. Departamento de Psicologia. Universidade Federal de São Carlos. Rodovia Washington Luis, Km235, Bairro Monjolinho, CEP 13565-905, São Carlos-SP, Brasil.E-mail: carmojs@gmail.com. 